\title{
Hétérogénéité des marchés et prix sans substance
}

Un commentaire de L'Empire de la valeur d'André Orléan (Seuil, 2011)

\section{Richard Arena}

\section{CpenEdition}

\section{Journals}

Édition électronique

URL : http://journals.openedition.org/ress/2308

DOI : $10.4000 /$ ress. 2308

ISSN : 1663-4446

Éditeur

Librairie Droz

\section{Édition imprimée}

Date de publication : 1 décembre 2012

Pagination : 218-224

ISBN : 978-2-600-01704-6

ISSN : 0048-8046

\section{Référence électronique}

Richard Arena, "Hétérogénéité des marchés et prix sans substance », Revue européenne des sciences sociales [En ligne], 50-2 | 2012, mis en ligne le 01 janvier 2016, consulté le 19 avril 2019. URL : http:// journals.openedition.org/ress/2308; DOI : 10.4000/ress.2308 
GOSSEN Hermann, 1995 (I854), Exposition des lois de l'échange et des règles de l'industrie qui s'en déduisent, trad. française, Paris, Économica.

HIRSCHMAN Albert, 1977, Les Passions et les intérêts. Justifications politiques du capitalisme avant son apogée, trad. française, Paris, PUF.

KARPIK Lucien, 2007, L'Économie des singularités, Paris, Gallimard.

MCMILLAN John, 1994, «Selling Spectrum Rights », Journal of Economic Perspectives, 8-3, p. $145-162$.

MAUDUIT Laurent, 2012, Les Imposteurs de l'économie. Comment ils s'enrichissent et nous trompent!, Paris, J.-C. Gawsewitch éditeur.

ORLEAN André, 1999, Le Pouvoir de la finance, Paris, Odile Jacob.

-, 2002, «Le tournant cognitif en économie», Revue d'économie politique, II2-5, p. $717-738$.

ROTH Alvin, 2010, «Experiments in Market design », in J. Kagel \& A. Roth, Handbook of Experimental Economics, vol. 2., à paraître.

STEINER Philippe, 2011, «The Creator, human conduct and the maximisation of utility in Gossen's economic theory», European Journal of the History of Economic Thought, 18-3, p. 353-379.

-, 2012, «Les performations économiques et axiologiques des échanges», conférence à l'Universidade Federal de Rio Grande do Sul, Gemass Working Paper, disponible sur le site du Gemass: http://www.gemass.fr

-, TRESPEUCH Marie, 2013, «La construction sociale de l'intérêt: les jeux d'argent en ligne et les organes à transplanter», Revue française de sociologie, à paraître.

\section{HÉTÉROGÉNÉITÉ DES MARCHÉS ET PRIX SANS SUBSTANCE Un commentaire de L'Empire de la valeur d'André Orléan (Seuil, 20II)}

par Richard Arena

GREDEG \& MSH, CNRS/Université de Nice-Sophia Antipolis arena.unice@gmail.com

L’ouvrage d'André Orléan - L’Empire de la valeur - présente deux caractéristiques. D’un côté, la publication de ce livre intervient dans une période où, selon l'auteur, «l'économie en tant que discipline traverse [...] une grave crise de légitimité» (EV, p. 9). La crise financière de 2008 aurait ainsi permis de mettre en lumière les erreurs et les limites de la théorie économique moderne et, en particulier, de celles qui sont liées à l'un de ses fondements essentiels, le concept de «valeur économique». D’un autre côté, l'ouvrage d’André Orléan offre au lecteur une synthèse à la fois convaincante et innovante de beau- 
coup des contributions fondamentales que l'auteur a lui-même consacrées par le passé à des thèmes de recherche tels que le mimétisme des comportements sur les marchés, la signification de la monnaie, le fonctionnement et l'efficience des marchés financiers ou encore l'importance des croyances collectives et des représentations conventionnelles en matière monétaire et financière. Cette synthèse a pour objet explicite de «refonder l'économie » (tel est même le sous-titre de l'ouvrage).

L'Empire de la valeur comprend trois parties. La première et, en particulier, ses deux premiers chapitres, sont largement critiques et interrogent les fondements des «théories de la valeur ». Les deuxième et troisième parties participent en revanche à cette tentative constructive de refondation de l'économie.

Les deux dernières parties du livre plaident pour une conception «institutionnaliste» (EV, p. I86) : selon le point de vue de l'auteur, «une véritable refondation de l'économie passe nécessairement par l’affirmation de son appartenance à part entière aux sciences sociales. Il faut défendre l'idée que le fait économique est un fait social comme les autres. Il ne possède en rien une essence particulière qui justifierait une épistémologie spécifique ou une discipline indépendante» (idem). Selon cette conception, l'économie doit adopter une approche «unidisciplinaire» dont l'objectif est de «surmonter les divisions artificielles que connaissent actuellement les sciences sociales, en affirmant leur profonde unité conceptuelle» (idem).

Cette conception unidisciplinaire de l'économie peut ainsi se construire en combinant les apports d'un certain nombre de penseurs en sciences sociales qui n'étaient pas des économistes ou qui, tout en l'étant, ont défendu une approche éloignée des théories de la valeur-substance: Durkheim, Simmel et Simiand ou Veblen, Knight et Keynes, par exemple. Elle repose fondamentalement sur «le désir de chacun à l'égard des biens liquides » (EV, p. I55). Or, ce désir ne dépend pas d’une qualité des biens posée a priori et fondée sur une substance mais relève d'une logique autoréférentielle qu'expriment notamment les comportements mimétiques. En effet, «c'est exclusivement via l’acquisition de biens liquides que l'individu marchand peut prétendre à la pleine reconnaissance de ses droits sur les marchandises. Dans une économie marchande, la puissance, c'est-àdire le pouvoir d’acheter, prend la forme d’une quantité : la quantité de biens liquides qui sont aux mains du producteur-échangiste, de telle sorte que le bien liquide est conduit naturellement à servir d'unité de mesure du pouvoir d'achat » (idem). En outre, ces quantités tendent à s'uniformiser avec l'évolution de l'économie marchande «autour d'une définition unique de la liquidité qui acquiert, ce faisant, une emprise extrême sur les acteurs 》 (EV, p. I6I). Tout le problème est alors que, si le désir à l'égard des biens liquides s'auto-entretient et se renforce, en revanche, cette tendance à l'uniformité est «plus imposée que désirée» (EV, p. 165) et est porteuse de résistances provenant de groupes privés défendant leurs seuls intérêts propres. C'est là précisément que se situe l'origine 
des crises monétaires, à travers «l'émergence d'une dissidence cherchant à faire prévaloir de nouvelles normes monétaires» (EV, p. I66).

Ces parties constructives de l'ouvrage abandonnent l'idée d'une valeur-substance préexistante à l'échange et défendent celle d'une valeur monétaire purement représentative reflétant les interactions sociales entre agents mais aussi selon l'auteur les «puissances collectives qui viennent dicter aux individus ce qu'il convient de faire» (EV, p. 324). Cette approche de la «refondation» de l'économie est certainement très stimulante: une grande partie de ses avancées permet de constituer de précieux matériaux dans la perspective de la construction d'une théorie économique plus soucieuse de rendre compte du «monde réel», tel quanalysé par les différentes sciences sociales, que de lui appliquer à tout prix et unilatéralement une axiomatique des choix rationnels. Il en est ainsi tout particulièrement pour ce qui concerne les analyses du couple monnaie / valeur et du fonctionnement des marchés financiers.

On peut toutefois s'interroger sur le degré de généralité de l’analyse proposée. Le poids de l'auto-référentialité est certainement essentiel sur des marchés tels que les marchés monétaires et financiers. En est-il autant des marchés pour lesquels le poids de la technologie est important, qu'il s'agisse des marchés industriels ou des marches de la connaissance? On peut d’autant plus se poser cette question que le livre d’André Orléan «porte exclusivement sur l'économie marchande et non sur le capitalisme», excluant ainsi d'emblée l'analyse du «rapport salarial» (EV, p. 23). Il nous semble en effet que, si l'activité de production (et en particulier de production capitaliste) était considérée en tant que telle, indépendamment de celle de l'échange (comme elle l'est, par exemple, dans le Traité de la Monnaie et dans les articles de Keynes de la fin des années 1930 autour du Motif de finance; dans Production de Marchandises par des Marchandises de Sraffa ou dans Une théorie évolutionniste du changement économique de Nelson et Winter), les choses prendraient cependant un autre tour. Il faudrait tout d'abord distinguer soigneusement ces deux activités et ne pas réduire la production à un échange entre produits et facteurs primaires. Il faudrait dès lors les articuler au sein d'une conception plus large de l'activité économique que celle de la sphère marchande, par exemple, en distinguant au moins les marchés des biens non produits (tels que les marchés financiers, les marchés d'occasion ou les marchés des facteurs primaires, etc.), les marchés de biens produits (tels que les marchés de marchandises reproductibles) et les marchés de la connaissance (tels que les marchés inter-entreprises visant à inventer de nouveaux biens ou de nouvelles technologies), ce que ne fait pas en général la théorie économique usuelle. Dans la perspective d'un recours au concept de surplus ou de surproduit, il faudrait également s'interroger sur la notion de coût et plus seulement sur celle de valeur ou de prix et distinguer ce qui relève du coût techniquement «nécessaire» et ce qui correspond à une représentation ad hoc du coût, liée à des règles ou des conventions sociales, alors même que le concept de productivité marginale exclut ce type de questionnement, comme on peut le lire dans les manuscrits de Sraffa: 
Il existe beaucoup de coûts socialement nécessaires qui sont présentés comme des nécessités techniques. Prenons le travail d'un contrôleur de billets sur un autobus ou un train: évidemment, le train roulerait tout aussi bien si aucun ticket n'était contrôlé; toutefois, si aucun des voyageurs ne payait sa place, les actionnaires obligeraient ce train à s'arrêter; ainsi le travail du contrôleur de billets empêche les actionnaires d'arrêter le train; les actionnaires seraient aussi efficaces que le manque de charbon lorsqu'ils arrêtent les trains. (Sraffa Papers, D3/12/18 [11], date de 1942).

Ce type de questionnement n’a évidemment pas de sens dans une économie purement marchande mais il est essentiel dans une économie capitaliste de marché.

Il faut en outre se poser la question de la répartition des revenus en fonction du rôle et des statuts des agents dans l'activité productive, sans supposer nécessairement a priori que les agents sont payés en fonction de leur contribution productive mesurée par leur productivité marginale. Il faut enfin construire une théorie du changement technologique, dans laquelle les notions d'état des techniques et de fonction de production n'ont plus de signification économique.

Or, toutes ces nécessités théoriques ne sont ni incompatibles avec l’analyse qu’André Orléan propose des marchés monétaires et financiers, ni avec la notion d’autoréférentialité ou celles de dépendance à l'égard du sentier et de rendement croissant d’adoption évoquées par l'auteur dans les chapitres 2 et 3 de son livre. Simplement, à notre avis, elles pourraient utilement compléter et enrichir sa construction, alors que l'exclusion de l'activité de production capitaliste de L'Empire de la valeur l'interdit. En outre, elles n'impliquent pas nécessairement le recours à une théorie de la valeur-substance fondée sur le travail, comme on pourrait éventuellement le penser.

L'ensemble de ces remarques n'est pas sans conséquences lorsque l'on cherche à situer la portée du texte d’André Orléan.

D’une part, le fait de choisir le contexte d'une économie purement marchande exclut d'emblée la prise en compte de ces dimensions productive et technologique qui ont pourtant joué un rôle essentiel dans la dynamique de moyenne et de longue période de notre système économique et sont largement ignorées par la théorie économique usuelle fondée sur la valeur-utilité.

D’autre part, il conduit à s'interroger sur certaines des conclusions de l'auteur, par exemple dans ses remarques à propos de l'analyse économique de Sraffa. Ainsi, il est inexact d'écrire que chez Sraffa, l'économie «reste une économie de troc puisque les biens s'y échangent exclusivement [souligné par nous] contre d'autres biens » (EV, p. 30), alors que rien n'est dit à propos du marché dans Production de Marchandises. La lecture des manuscrits de Sraffa consultables à la Wren Library de Cambridge nous montrent cependant comment, pendant toute sa vie, l'importance de la monnaie, du crédit et des marchés financiers a été essentielle pour cet auteur (voir notamment Panico, 200 I, de Cecco, 2008 et Arena, 20Io) sans pour autant le conduire à rejeter toute théorie de 
la représentation sociale des prix relatifs. Simplement et contrairement à ce que la tradition néo-ricardienne a souvent prétendu, Sraffa ne fait pas reposer ces prix sur «la loi de la gravitation» (EV, p. 35 ; voir notamment Dupertuis et Sinha, 2009, Sinha, 2010 et Blankenburg, Arena et Wilkinson, 2012). Il distingue en outre la question de la représentation des prix relatifs (qui est fondée sur une vision synthétique et socialement spécifiée des activités de production et de répartition du surplus analogue à ce que Wittgenstein, proche de Sraffa, appelait le «tableau synoptique» d'une «forme de vie » ou «d'activité »; voir Arena, 20I2) de celle de la réalisation des prix monétaires. Cette démarche exclut toute référence à une théorie de la valeur-substance et ne saurait donc se situer «dans la perspective de la valeur-travail» (EV, p. 39) que Sraffa rejeta toute sa vie avec constance et contribua puissamment à exclure du corpus de la tradition classique (Steedman, 1977). Elle ne fait pas référence, malgré les apparences, à «une concurrence élargie au capital et au travail» (EV, p. 39) qui justifierait l'uniformité du taux de profit (Arena, 2012; Sinha, 20ıo). Ainsi, l’inclusion de la théorie de Sraffa au sein des théories de la valeur-substance qui rejettent le rôle essentiel de la monnaie ou des marchés financiers ne nous parait pas fondée.

De même, on s'interrogera sur l'idée selon laquelle la représentation typique du fonctionnement des marchés fondée sur la valeur-utilité reste aujourd'hui celle de la théorie de l'équilibre économique général (EEG) supposée impliquer une analyse de la valeur-substance. Depuis la remise en cause complète de ce paradigme dans les années 1970 (à la suite, notamment, des articles de Debreu, Mantel et Sonnenschein) puis dans les années 1980 (après la publication de l'ouvrage de Franklin Fisher; voir EV, p.69) la représentation des marchés qui prédomine au sein de la théorie économique usuelle dépend toujours davantage du type de marche privilégié : à titre d'exemple, nous savons que ce sont les applications de la théorie des jeux - et non de la théorie de l'EEG - qui constituent aujourd'hui le fondement de la représentation la plus utilisée des marchés oligopolistiques et que celle-ci est le plus souvent construite à partir de la notion d'équilibre partiel.

En outre, le concept de valeur-substance semble de moins en moins présent au sein du paradigme de la théorie économique usuelle. On se réfèrera à ce propos à la contribution de Gioccoli (2005). Le point de vue défendu dans cette contribution est précisément que l'économie a connu une transformation radicale de son image depuis ses origines, ce qui n’a pas été sans effets sur le corps des connaissances qui la constitue. Selon l'auteur, l'économie est ainsi passée d'une représentation de «systèmes de forces » à une représentation de «systèmes de relations ». Lorsque l'économie est un système de forces, l'objet d'étude est l'analyse des processus conduisant à l'équilibre. En revanche, lorsque l'économie est un système de relations, l'objet d'étude est l'analyse des conditions d'existence de l'équilibre. Cette distinction est appliquée par Gioccoli pour opposer rationalité comme maximisation de l'utilité et rationalité comme cohérence des choix. 
Ainsi, décrivant la manière dont l'économie «néo-classique » contemporaine caractérise la notion de rationalité, Gioccoli note que l’agent rationnel «est un décideur bayésien qui formule des anticipations rationnelles à l'instar d'un expert en économétrie lorsqu'il s'agit pour lui de choisir entre les configurations d'un équilibre général multipériodique et privilégie les stratégies correspondant à un équilibre de Nash lorsqu'il est confronté à des situations d'interaction entre agents» (Giocoli, 2005, p. 202). À nouveau et pour le dire autrement, la caractéristique dominante du choix rationnel renvoie donc aujourd'hui principalement à la capacité de l'agent économique à réagir de manière cohérente aux incitations qui lui sont proposées plus qu'à son désir d'obtenir la maximisation sous contrainte d'un montant d'utilité-substance. Cette remarque est d'autant moins surprenante qu'elle trouve déjà son origine chez Pareto et qu'elle n’a jamais cessé depuis de se développer et de se généraliser au sein de la tradition néo-classique. On comprend alors pourquoi, au plan théorique, les économistes venus de la tradition néo-classique ont souvent tourné définitivement la page de la théorie de l'EEG au profit de l'économie de l'information, de l'économie comportementale, ou pour reprendre l'énumération critique d’André Orléan (EV, p. 9), «des équilibres multiples, de l'économie expérimentale, voire de la neuroéconomie». Le point de vue commun de ces approches centrifuges ne repose plus tellement sur l'idée que le marché est le thème de recherche central des économistes et que la substance-utilité est le fondement de l'analyse de ce dernier. Il est plutôt dans la recherche d'une formalisation logique compatible avec une justification non exclusivement déductive d'une rationalité économique s'appliquant à des contextes divers, y compris non-marchands.

Dans cette perspective, s'il est exact d'écrire quau sein de la TEEG, «la préférence ne dépend en rien de ce que font les autres : ni de ce qu'ils consomment, ni ce qu'ils désirent» (EV, p. 56), en revanche cette remarque n'est plus valide en théorie des jeux par exemple. Ainsi, la version de cette théorie fondée sur l'altruisme ou la sympathie repose précisément sur une préférence dépendante des comportements des autres (Sobel, 2005). Il conviendrait ainsi d'opérer un véritable tour d'horizon de la théorie économique usuelle, désormais moins unifiée que par le passé, et de mesurer ses différents apports à l'analyse de la valeur économique afin de mieux apprécier l'impact de l'ouvrage d’André Orléan. Il est cependant évident que, loin de remettre en cause les avancées substantielles développées dans les parties constructives de L’Empire de la valeur, ce bilan permettrait de mieux mesurer quelles sont les différentes parties de la théorie économique usuelle qu'il convient aujourd'hui d'abandonner. 


\section{Références}

ARENA R., 2010, «Corporate limited liability and Cambridge economics in the inter-war period: Robertson, Keynes and Sraffa », Cambridge Journal of Economics, 34-5, September, p. 869-883.

-, 2012, «Sraffa and Wittgenstein on snapshots and forms of life» in R. Ciccone, M. A. Campus, S. Levrero et A. Stirati (eds): Sraffa's Production of Commodities by Means of Commodities 1960-2010, Critique and reconstruction of economic theory, Londres-New York, Routledge, à paraître.

BLANKENBURG S., ARENA R., WILKINSON F., 20I2, «Introduction 》 to the special issue on «Sraffa and the Unpublished Manuscripts», Cambridge Journal of Economics, 36-6, à paraître.

DE CECCO M., 2008, «Sraffa's Lectures on Continental Banking» in H. Kurz, L. Pasinetti, N. Salvadori (eds), Piero Sraffa: The Man and the Scholar - Exploring His Unpubllished Papers, Londres-New York, Routledge, p. 185-194.

DUPERTUIS M., SINHA A., 2009, «A Sraffian Critique of the Classical Notion of Centre of Gravity», Cambridge Journal of Economics, 33-6, p. 1065-1087.

GIOCCOLI N., 2005, «Modeling rational agents: the consistency view of rationality and the changing image of neoclassical economics», Cahiers d'économie politique, 49, p. $177-208$.

PANICO C., 200I, «Monetary analysis in Sraffa's writings» in T. Cozzi. et R. Marchionatti (eds), Piero Sraffa's Political Economy, A Centenary Estimate, Londres-New York, Routledge, p. 285-310.

SINHA A., 2012, «Listen to Sraffa's silences: A new interpretation of Sraffa's Production of Commodities», Cambridge Journal of Economics, 36-6, à paraître.

SOBEL J., 2005, «Interdependent Preferences and Reciprocity», Journal of Economic Literature, American Economic Association, 43-2, June, p. 392-436.

SRAFFA P., 1942, Sraffa Papers, D3/I2/18, (II), Trinity College, Cambridge, Wren Library. STEEDMAN I., 1977, Marx After Sraffa, Londres, NLB. 